\title{
Targeted gene therapy of nasopharyngeal cancer in vitro and in vivo by enhanced thymidine kinase expression driven by human TERT promoter and CMV enhancer
}

\author{
Cong-Xiang Shen, Zhong Wen", Yu-Hong Qian, Shao-Feng Mu, Xiao-Fang Guan
}

\begin{abstract}
Background/Aim: To explore the therapeutic effects of thymidine kinase (TK) expressed by enhanced vector pGL3basic- hTERTp-TK-EGFP-CMV driven by human telomerase reverse transcriptase promoter (hTERTp) as well as cytomegalovirus immediate early promoter enhancer (CMV).

Materials/Methods: Enhanced TK-EGFP expression was confirmed by fluorescent microscopy, real time PCR and telomerase activity. Its effects were examined by survival of tumor cells NPC 5-8F and MCF-7, index of xenograft implanted in nude mice and histology.

Results: Compared with non-enhanced vector pGL3-basic-TK-hTERTp-EGFP, TK expressed by the enhanced vector significantly decreased NPC 5-8F and MCF-7 cell survival rates after ganciclovir (GCV) treatment $(p<0.001)$ and tumor progress in nude mice with NPC xenograft and treated with GCV, without obvious toxicity to mouse liver and kidney.
\end{abstract}

Conclusion: The enhanced TK expression vector driven by hTERTp with CMV enhancer has brighter clinical potentials in nasopharyngeal carcinoma therapy than the non-enhanced vector.

\section{Introduction}

Nasopharyngeal carcinoma (NPC) is one of highly prevalent, most harmful malignant tumors in Southern China and Southeast of Asia. It is caused by the interaction between genetic background and environmental factors such as Epstein-Barr virus. At present, radiotherapy and/or induction chemotherapy is the mainstay of treatment modalities. Despite continuously progress in radiotherapeutic equipment and technology, the 5-year survival rate of NPC remains about $50 \%$ without fundamental improvement over the past several decades. Understanding the etiology and developing new effective therapeutic modality are particularly important in NPC treatment.

Suicide gene therapy is a promising modality for cancer treatment. Such therapy introduces a drug

\footnotetext{
* Correspondence: wenzhong60@163.com

Otolaryngology-Head \& Neck Surgery, Zhujiang Hospital, The Southern Medical University, Guangzhou 510282, China
}

susceptible gene such as herpes simplex virus thymidine kinase (TK) gene into tumor cells. Expressed TK phosphorylates its substrate, a nontoxic prodrug ganciclovir (GCV), leading to accumulation of the toxic ganciclovir triphosphate and cell apoptosis. The ideal suicide gene expression constructs should have high specificity and killing efficacy to tumor cells. To selectively introduce suicide gene into tumor cells, many tumor specific promoters have been employed to construct tumor-specific suicide gene expression vectors.

Human telomerase reverse transcriptase (hTERT), the core component of telomerase, plays important roles in vast majority of malignant tumors including nasopharyngeal carcinoma. The telomerase activity and level of hTERT expression are enhanced in all nasopharyngeal carcinoma cell lines and 88\% nasopharyngeal tissues. Their activities are closely correlated with clinical biological characteristics of nasopharyngeal carcinoma[1,2]. Therefore, telomerase/hTERT is utilized as a targeted
C Biomed Central

(c) 2010 Shen et al; licensee BioMed Central Ltd. This is an Open Access article distributed under the terms of the Creative Commons Attribution License (http://creativecommons.org/licenses/by/2.0), which permits unrestricted use, distribution, and reproduction in any medium, provided the original work is properly cited. 
gene for treatment of nasopharyngeal carcinoma and its promoter has been widely employed to drive the tumorspecific expression of exogenous genes. For example, Wang et al[3] and Zhang et al [4] constructed vectors pGL3-hTp-TK/GCV and TERT-E1A-TK, respectively, both of which can kill lung cancer cells and transplanted tumor in vitro and in vivo. Zheng et al [5] constructed vector $\mathrm{pHSV}-\mathrm{TK} / \mathrm{CRAD}$, which can significantly enhance the killing effect of GCV on liver cancer in animal. Shen et al [6] selectively expressed shRNA in nasopharyngeal carcinoma cells by introducing hTERT, which successfully inhibited telomerase activity and induced cell apoptosis. We [7] have reported previously that administration of antisense oligodeoxynucleotide of telomerase RNA (hTR) and hTERT subunit can inhibit telomerase in tumor cells and induce tumor cell apoptosis. Recently, we $[8,9]$ exploited the hTERT promoter to construct pGL3-hTERTp-TK vector and introduced the vector into NPC tumor cells in vitro and in vivo in mice xenograft, which killed NPC tumor cells and xenograft without observing toxicity to liver and kidney. However, its tumor killing efficacy is less than that of non-TK targeted system driven by cytomegalovirus immediate early promoter (CMV). To further increase TK mediated tumor killing efficacy and facilitate tracing TK expression, we constructed a new vector by inserting a CMV enhancer and an EGFP reporter gene into pGL3hTERTp-TK vector, and evaluated its therapeutic efficacy in in vitro and in vivo tumor therapy.

\section{Materials and methods 1. Reagents}

Fetal bovine serum (FBS) was purchased from Hangzhou Sijiqing Company. PCR kit and TaqMan real time PCR kit were from Takara Bio-engineering Co., Ltd. 3(4,5-Dimethylthiazol-2-yl)-2,5-diphenyltetrazolium bromide (MTT) was from Sigma, USA; Ganciclovir (GCV) was from ROCH company. Lipofectamine 2000, DMR IE2C and Trizol were from Invitrogen. TRAPEZE ${ }^{\bullet} \mathrm{RT}$ telomerase activity detection kit was purchased from KeyGen (Nanjing, China). Plasmid Midi Kit was from Heda Biotech (Guangzhou, China). All PCR primers were synthesized by Shanghai Ying-Jun Biotechnology Co., Ltd.

\section{Cell lines}

Human nasopharyngeal carcinoma 5-8F cells (NPC 5$8 \mathrm{~F}$ ), human breast cancer cells MCF-7 and human vascular endothelial cells ECV were kindly provided by Department of Cell Biology, the Southern Medical University, and maintained in RPMI 1640 supplemented with $10 \%$ heat-inactivated fetal bovine serum at $37^{\circ} \mathrm{C}$ in a $5 \% \mathrm{CO}_{2}$ incubator (Shell LAB, USA) as previously reported [10].

\section{Construction of plasmid with luciferase reporter gene} EGFP gene was obtained from pEGFP-N1 by PCR using forward primer Egfp-F: CCCAAGCTTATGGTGAGCAAGGGCGAGGAG and reverse primer Egfp-R: GCTCTAGATTACTTGTACAGCTCGTCCATGC. 406 bp CMV enhancer fragment was obtained from pEGFP$\mathrm{N} 1$ by PCR using forward primer hCMVen-F: 5CGGGATCCCGCGTTACATAACTTACGGT-3' and reverse primer hCMVen-R: 5-ACGCGTCGACCAAAACAAACTCCCATTGAC-3. 1131 bp TK gene with NCBI accession number AY575228 was obtained from pMD18TK by PCR using forward primer 5-CCGCTCGAGATGGCTTCGTACCCCTGC-3' and reverse primer 5CCCAAGCTTGTTAGCCTCCCCCATCTC-3. The 260 bp hTERT promoter was obtained from pMD18T-hTERTp using forward primer hTERTp-F: 5GGGGTACCAGTGGATTCGCGGGCACAGACG-3' and reverse primer hTERTp-R: 5-CCGCTCGAGAGGGCTTCCCACGTGCGCAGCA-3. All PCR fragments were verified by $\mathrm{DN}$

A sequence analysis. Stop codon TGA of TK gene was removed in $\mathrm{TK}$ reverse primer to facilitate the construction of TK-EGFP fusion protein. EGFP fragment was digested with Hind III and Xba I and subcloned into pGL3basic plasmid to obtain pGL3-basic-EGFP. TK fragment was excised with Hind III and Xho I and subcloned into pGL3-basic-EGFP to construct pGL3-basic- TK-EGFP. hTERTp fragment was subcloned into pGL3-basic-TK-EGFP at Kpn I and Xho I sites to construct pGL3basic-TK-hTERTp-EGFP. CMV enhancer fragment was inserted into pGL3-basic-TKhTERTp-EGFP at BamH I and Sal I site according to previous reports $[11,12]$ to construct the enhanced vector pGL3-basichTERTp-TK- EGFP-CMV. All plasmids were verified by restriction enzyme digestion.

\section{Cell transfection as well as expression of TK-EGFP fusion protein}

$1 \times 10^{6}$ NPC 5-8F, MCF-7 and ECV cells at logarithmic phase were inoculated into one well of 6-well plate with duplications, respectively. 12 hours after inoculation, cells at about $80 \%$ confluency were transfected with 4 $\mu \mathrm{g}$ of plasmid pGL3-basic-hTERTp-TK-EGFP-CMV or pGL3-basic-hTERTp-TK-EGFP by mixed with $4 \mu \mathrm{l}$ Lipofectamine 2000 according to the protocol provided by the manufacturer. 24 hours after transfection, the expression of TK-EGFP fusion protein was directly observed with fluorescent microscopy (Nikon Eclipsete 2000-U, USA). 


\section{RNA Isolation and TK mRNA level detection by quantitative real-time PCR}

48 hours after transfection, total RNA was extracted with Trizol (Invitrogen) following the manufacturer's instruction. $4 \mu \mathrm{L}$ mRNA of each sample was used as template in quantitative real-time PCR performed in an ABI 7500 Real-Time PCR system using Taqman PCR kit based on the manufacturer's protocol. The specific primers used in these reactions were followings: TK forward 5'-AGCAAGAAGCCACGGAAGTC-3' and reverse 5'-AGTTGCGTGGTGGTGGTTTT-3'; human $\beta$-actin forward 5'-GCATGGGTCAGAAGGATTCCT-3' and reverse $5^{\prime}$-TCGTCCCAGTTGGTGACGAT- $3{ }^{\prime}$. Relative levels of TK gene expression were normalized to $\beta$-actin mRNA level.

\section{Telomerase activity measurement}

NPC 5-8F cells at logarithmic phase were inoculated into three wells of a 6 -well plate with $1 \times 10^{6} /$ well. Twelve hour later, two wells of cells were transfected with $8 \mu \mathrm{g}$ pGL3-basic- hTERTp-TK-EGFP-CMV plasmid. Twelve hours after transfection, one well of cells transfected with pGL3-basic-hTERTp-TK-EGFP-CMV were treated with $10 \mu \mathrm{g} / \mathrm{mL}$ GCV. 48 hours after drug treatment, telomerase activities of all three well of cells were measured using PCR-based TRAP telomerase activity detection kit. As control, telomerase activity of 1 $\times 10^{6}$ ECV cells at logarithmic phase was also detected using the same method. The PCR products were separated on $12 \%$ non-PAGE and visualized by silver stain.

\section{Cell survival rate measurement by MTT method}

NPC 5-8F cells at logarithmic phase were inoculated into 15 wells of 96 -well plate with $1 \times 10^{5}$ cells in each well. Twelve hours later, 3 wells of NPC 5-8F cells were used as blank, 3 wells were transfected with $2.4 \mu \mathrm{g}$ pGL3-basicEGFP as control, 6 wells were transfected with pGL3basic- hTERTp-TK-EGFP-CMV. Twelve hours after transfection, control group and three wells of the cells transfected with pGL3-basic-hTERTp-TK-EGFP-CMV were treated with $10 \mu \mathrm{g} / \mathrm{mL}$ GCV. 72 hours after treatment, all cells were subjected to MTT assay as described previously [10]. In detail, $20 \mu \mathrm{l}$ of $5 \mathrm{~g} / \mathrm{L} \mathrm{MTT}$ solution was added into each well of the 96-well plate, and the plate was incubated for 4 hours at room temperature. After the culture solution was removed, $150 \mu \mathrm{l}$ DMSO was added into each well and oscillated for 10 minutes. Then the absorption at $570 \mathrm{~nm}$ was measured with Startfax 2100 microplate reader (USA). Cell survival rate was expressed as A/ $\mathrm{B} \times 100 \%$, where A was the absorption from cells transfected with plasmid and treated with or without GCV, and $\mathrm{B}$ was that from the blank group.

The same experiment was performed using MCF-7 cells instead of NPC 5-8F cells.

\section{In vivo animal experiments}

Healthy male and female nude BALB/c nu/nu mice of age 4-5 weeks, weighing between 18-22 g, were from the Experimental Animal Centre of The Southern Medical University, and maintained in a SPF level aseptic environment. The animals were free access to aseptic rodent diet and water. The protocol of animal experiments was approved by ethical and humane committee of Zhujiang Hospital, The Southern Medical University.

NPC 5-8F cells at logarithmic phase were prepared as $5 \times 10^{6}$ cells $/ \mathrm{mL}$ single cell suspension in phosphate buffered saline (PBS) and $0.2 \mathrm{ml}$ of cell suspensions were subcutaneously inoculated into the left flank of $\mathrm{BALB} / \mathrm{c}$ nude mice. The cancer growth was monitored every 3 days starting at the day after inoculation by calipers to record the length (a) and width (b), and tumor volume were calculated by the formula $V=1 / 2\left(a \times b^{2}\right)$. When majority tumors reached $1.2 \sim 1.5 \mathrm{~cm}$ in diameter at day 10 after inoculation, nude mice were randomly divided into 6 groups: blank group, Lipofectamine group, non-enhanced group, enhanced group, enhanced/GCV group, and GCV group. Mice in blank and GCV groups were intratumorally injected with PBS; mice in Lipofectamine group were intratumorally injected $25 \mu \mathrm{L}$ Lipofectamine alone; mice in nonenhanced group were intratumorally injected with mixture of $25 \mu \mathrm{L}$ Lipofectamine with $10 \mu \mathrm{g}$ plasmid pGL3basic-hTERTp-TK-EGFP; mice in enhanced and enhanced/GCV groups were injected with the mixture of $25 \mu \mathrm{L}$ Lipofectmine 2000 and $10 \mu \mathrm{g}$ plasmid pGL3basic-hTERTp-TK-EGFP-CMV. All injections were performed repeatedly at the days 4, 7, 10 and 14 after the first injection. Meanwhile, mice in GCV and enhanced/ GCV groups were intraperitoneally injected $100 \mathrm{mg} / \mathrm{kg}$ bodyweight GCV every 2 days starting at day 1 after the first injection of the mixture for total 12 times. When the tumor volume reached $6 \mathrm{~cm}^{3}$ in mice from blank group, all mice were sacrificed by cervical dislocation and the whole tumors were removed and weighed, and livers and kidneys from mice in Lipofectamine, enhanced/GCV and GCV groups were preserved for further histopathological examination. The inhibition rate of different treatment on tumor growth was calculated according to the following formula:

Tumor inhibition rate $(\%)=($ tumor weight in non - treatment group - tumor weight in treatment group $) /$ tumor weight in non - treatment group $\times 100$

\section{Histopathological examination}

The preserved livers and kidneys were fixed with $10 \%$ formaldehyde solution and the sections were stained with hematoxylin and eosin, and analyzed by light microscopy. 


\section{Statistical analysis}

Data were analyzed with SPSS11.0 statistical software and expressed as mean \pm standard deviation. Statistical significant was analyzed using one-way ANOVA and $\mathrm{q}$ test. A p value less than 0.05 was considered as statistical significance.

\section{Results}

1. Identification of pGL3-basic- hTERTp -TK-EGFP-CMV plasmid

We constructed an enhanced TK expression vector as described in the Materials and Methods section. First we examined whether we successfully constructed the enhanced TK expression vector. Digestion with BamH I and Sal I, Xho I and Xba I, Kpn I and Hind III resulted in $406 \mathrm{bp}, 1850 \mathrm{bp}$ and $1400 \mathrm{bp}$ fragments, respectively, as expected. The sequences of TK gene, hTERTp and CMV enhancer have been confirmed by direct DNA sequences.

\section{Fluorescent level of TK-EGFP gene expression}

Then we measured the fluorescent level of TK-EGFP gene expression in NPC 5-8F and MCF-7 cells transfected with either the enhanced plasmid pGL3-basichTERTp-TK-EGFP-CMV or the non-enhanced pGL3basic-hTERTp-TK-EGFP by observing the fluorescent intensity of co-expressed GFP under fluorescent microscope. As shown in Figure 1, NPC 5-8F and MCF-7 cells transfected with the enhanced plasmid showed very strong green fluorescence (Figure 1a and 1b). NPC 5-8F cells transfected with the non-enhanced plasmid also had very strong green fluorescence (Figure 1c). However, compared with cells transfected with the enhanced plasmid, the fluorescent intensity was decreased. ECV cells transfected with the enhanced plasmid only showed weak, flurry fluorescence (Figure1d) under the same condition. Since the expression of TK-EGFP was controlled by hTERT promoter, therefore it was only expressed in telomerase-positive cells. Furthermore, TK was fused to EGFP, expression level of EGFP not only reflected the transfection efficient, but also indirectly indicated the relative expression level of TK.

\section{Enhanced TK mRNA level in cells transfected with pGL3-basic-hTERTp-TK- EGFP-CMV}

We further quantitatively examined the expression of TK gene in NPC 5-8F and MCF-7 cells at mRNA level by real-time PCR. Figure 2 showed the amplification curves of housekeeping gene ( $\beta$-actin and TK gene, and Table 1 showed the relative expression level of TK gene to $(\beta$-actin gene. TK gene expression in NPC $5-8 \mathrm{~F}$, MCF-7 and ECV cells transfected with the enhanced plasmid was 4.2-fold, 2.5-fold, and 0.0027-fold of $\beta$ actin, respectively. By contrast, the TK expression level in NPC 5-8F cells transfected with pGL3-basichTERTp-TK-EGFP was only 0.82 -fold of $\beta$-actin. No TK expression was detected in NPC 5-8F cells transfected with pGL3-basic-EGFP as expected. These results are consistent with that of Figure 1.

\section{Reduced telomerase activity by pGL3-basic-hTERTp-TK- EGFP-CMV/GCV}

Next we examined telomerase activity in PNC 5-8F cells transfected with the enhanced plasmid with or without GCV treatment. NPC 5-8F cells transfected with the enhanced plasmid were telomerase activity positive. However, the telomerase activity was decreased by 48 hours of GCV treatment. As control, ECV cells showed weak telomerase positive (Figure 3).

\section{Decreased survival rate of tumor cells transfected with the enhanced plasmid and treated with GCV}

Having confirmed that transfection of the enhanced plasmid increased the expression of TK, we further studied whether transfection of the enhanced plasmid could affect the effect of GCV on the survival rate of nasopharyngeal carcinoma NPC 5-8F cells and breast cancer MCF-7 cells by using MTT method. As shown in Tables 2 and 3, compared with non-transfected, untreated cells,
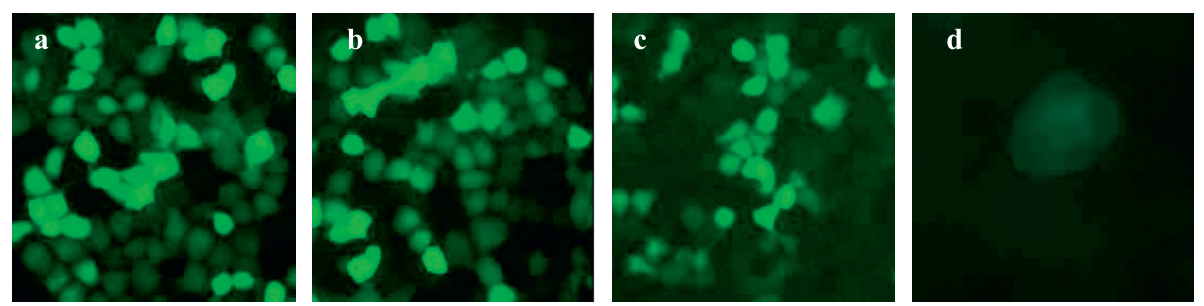

Figure 1 TK gene expression detected with fluorescent microscopy. Shown here are the cells 24 hours after transfection under fluorescent microscope (×100). (a) NPC 5-8F cells transfected with pGL3-basic-hTERTp-TK-EGFP-CMV; (b) MCF-7 cells transfected with pGL3-basic-hTERTp-TKEGFP-CMV; (c) NPC 5-8F cells transfected with pGL3-basic-TRETp-TK-EGFP; (d) ECV cells transfected with pGL3-basic-hTERTp-TK- EGFP. 


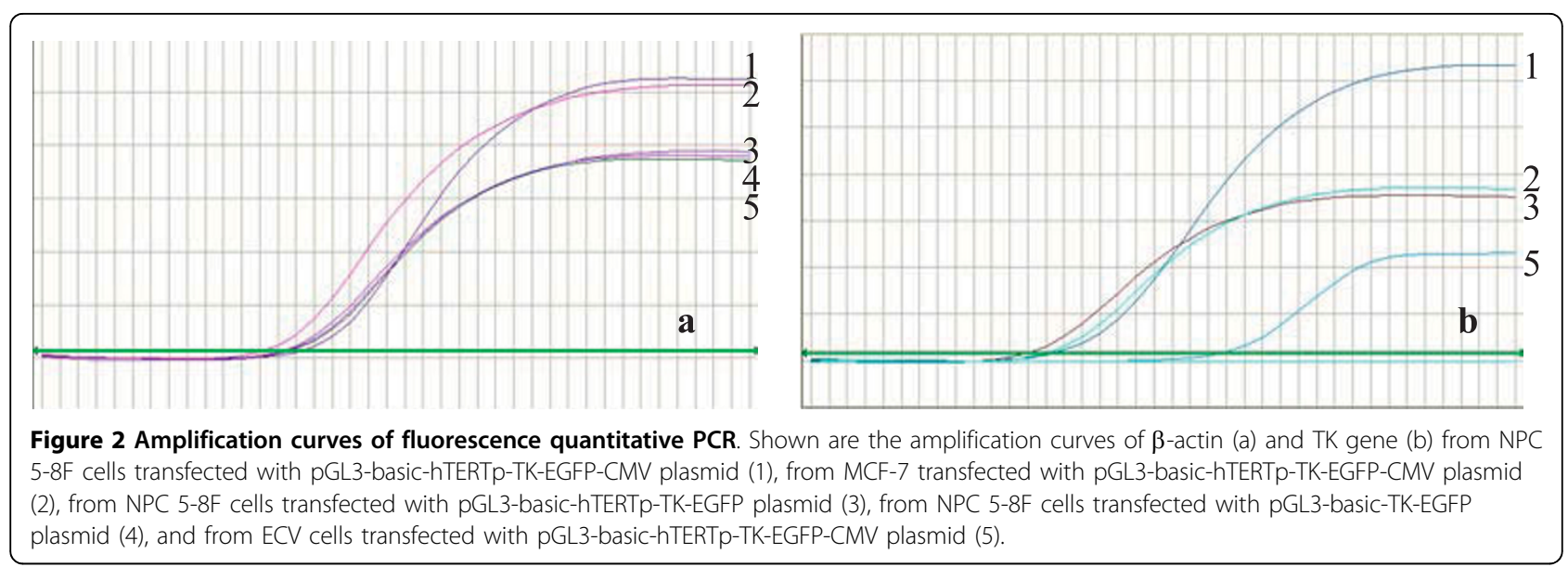

transfection of control plasmid pGL3-basic-EGFP had no effect on survival rates of tumor cells 5-8F and MCF-7 with GCV treatment, and transfection of the enhanced plasmid pGL3-basic- hTERTp-TK-EGFP-CMV alone did not change the survival rates of tumor cells NPC 5-8F and MCF-7. However, after GCV treatment, survival rates of NPC 5-8F and MCF-7 cells transfected with the enhanced plasmid decreased to $0.370 \pm 0.024$ and 0.462 \pm 0.049 , respectively, which was significantly lower than that, $0.533 \pm 0.020$ and $0.515 \pm 0.025$, of tumor cells NPC 5-8F and MCF-7 transfected with the plasmid pGL3basic-hTERTp-TK- EGFP and treated with GCV, respectively.

\section{Injection of pGL3-basic-hTERTp-TK-EGFP-CMV/GCV inhibited tumor progress in vivo}

Then we explored whether injection of pGL3-basichTERTp-TK-EGFP -CMV/GCV could inhibit tumor progress. As showed in Figure 4 and table4, nude mice inoculated NPC 5-8F cells developed tumor with volume

\section{Table 1 Expression of TK gene detected with real-time}

\section{PCR}

\begin{tabular}{llll}
\hline Sample & $\begin{array}{l}\text { Copy number } \\
\text { ( } \boldsymbol{\beta} \text {-actin) }\end{array}$ & $\begin{array}{l}\text { Copy number } \\
\text { (TK) }\end{array}$ & Relative folds to $\boldsymbol{\beta}$-actin \\
\hline 1 & $6.67 \mathrm{E}+07$ & $2.78 \mathrm{E}+08$ & $4.16792^{*}$ \\
2 & $4.50 \mathrm{E}+07$ & $1.13 \mathrm{E}+08$ & $2.51111^{* *}$ \\
3 & $7.76 \mathrm{E}+07$ & $2.17 \mathrm{E}+05$ & 0.00279639 \\
4 & $8.21 \mathrm{E}+07$ & Undetermined & Undetermined \\
5 & $1.69 \mathrm{E}+08$ & $1.39 \mathrm{E}+08$ & 0.822485 \\
\hline
\end{tabular}

Numbers 1, 2, 3, 4, 5 correspond to the numbers in Figure 3.

1: NPC 5-8F cells transfected with pGL3-basic- hTERTp-TK- EGFP- CMV;

2: MCF-7 transfected with pGL3-basic- hTERTp-TK- EGFP- CMV;

3: NPC 5-8F cells transfected with pGL3-basic- hTERTp-TK- EGFP;

4: NPC 5-8F cells transfected with pGL3-basic -TK-EGFP;

5: ECV cells transfected with pGL3-basic- hTERTp-TK- EGFP- CMV.

Data are presented as mean \pm standard deviation from these experiments. ${ }^{*} \mathrm{P}$ $<0.0001$ for sample 1 vs sample 3 , sample 1 vs sample 5 and sample 2 vs sample $3 .{ }^{* *} \mathrm{P}<0.001$ for sample 2 vs sample 5 . of $6.23 \pm 0.04 \mathrm{~cm}^{3}$ and weight of $2.68 \pm 0.02 \mathrm{~g}$. After injection of non-enhanced plasmid and GCV, the tumor volume and weight decreased to $3.51 \pm 0.02 \mathrm{~cm}^{3}$ and $1.51 \pm 0.01 \mathrm{~g}(\mathrm{p}=0.000)$, respectively. In comparison, after injection of the enhanced plasmid and GCV, the tumor volume and weight decreased to $2.27 \pm 0.02 \mathrm{~cm}^{3}$ and $1.17 \pm 0.01 \mathrm{~g}$, respectively, which were significantly lower than those of nude mice injected with the nonenhanced vector $(p=0.000)$. The inhibition rates of tumor progress were $43.68 \%$ and $56.34 \%$ for injection of non-enhanced and enhanced plasmids, respectively.

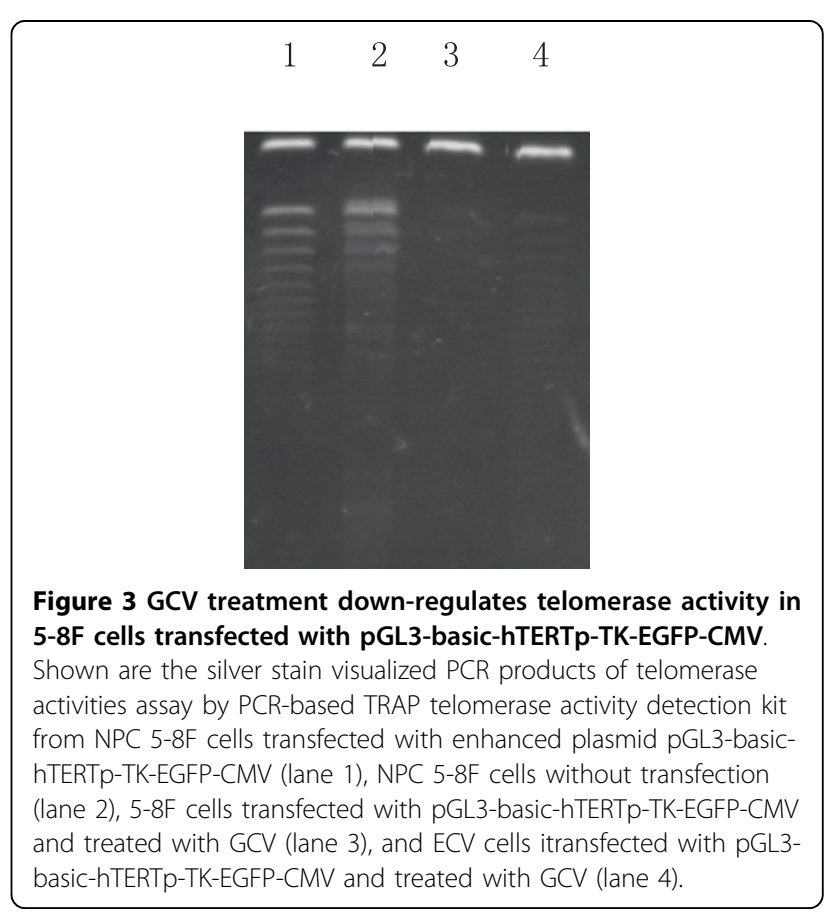


Table 2 PNPC cell survival rates measured by MTT assay

\begin{tabular}{ll}
\hline Codes and Samples & Survival rates \\
\hline A. Cells without treatment & 1 \\
B. Cells transfected with pGL3-basic-EGFP and with GCV treatment & $0.984 \pm 0.009$ \\
C. Cells transfected with pGL3-basic- hTERTp-TK-EGFP-CMV and treated with GCV & $0.370 \pm 0.024^{*}$ \\
D. Cells transfected with pGL3-basic-hTERTp-TK-EGFP-CMV without GCV & $0.982 \pm 0.010$ \\
E. Cells transfected with pGL3-basic-hTERTp-TK-EGFP and treated with GCV & $0.533 \pm 0.020^{*}$ \\
\hline
\end{tabular}

Data are expressed as mean \pm standard deviation from three experiments. ${ }^{*}$ indicates $p<0.0001$ compared with other groups

7. Injection of pGL3-basic-hTERTp-TK-EGFP-CMV/GCV had no toxicity to liver and kidney of nude mice

We further examined whether injection of GCV and the enhanced plasmid could have any toxicity to nude mouse. No obvious damages were observed in $\mathrm{H} \& \mathrm{E}$ stain in the livers and kidneys from nude mice in GCV/ enhanced, GCV and Lipofectamine 2000 groups.

\section{Discussions}

Molecularly targeted therapy is a promising research area in cancer therapy. Application of suicide gene in tumor therapy was limited due to lack of selectivity. Suicide gene TK or CD expression system driven by tumorspecific promoter has overcome the disadvantage and become a powerful modality in cancer therapy.

Identification of molecular targets is the key in molecularly targeted therapy. Molecules involved in carcinogenesis, cancer gene mutation, tumor angiogenesis and tumor signal transduction, telomerase, and growth factors such as epidermal growth factor are potential targets for tumor treatment. Gene mutation [13], EB virus [14], telomerase [1] and nasopharyngeal cancer stem cells $[10,15,16]$ are reportedly involved in the progress of nasopharyngeal cancer. Therapies targeted to the molecules and molecules related to those mentioned above have made primary progress in nasopharyngeal cancer treatment $[14,8,10]$. We have found that introduction of TK expression vector driven by hTERT promoter (hTERTp/TK) could kill nasopharyngeal carcinoma cells, nasopharyngeal carcinoma stem cells, and nasopharyngeal tumor xenograft in nude mice without side effects on cultured normal cells and damaging mouse liver and kidney functions [17]. Studies on other tumors also confirmed the efficacy of hTERTp/TK for cancer therapy. Introduction of herpes simplex TK gene expression virus vector driven by hTERT promoter (AdhTERT/TK) can specifically kill the undifferentiated thyroid tumor and thyroid tumor xenograft in nude mice, enhance the tumor GCV sensitivity without toxic reaction in liver and the whole body examined by liver pathology and serum enzymology [18]. By contrast, introduction of TK gene expression vector driven by CMV promoter (CMV/TK) not only kills tumor xenograft, but also demonstrates obvious liver pathological changes and damaged liver function revealed by serum enzymology. In addition, hTERT promoter has been used to target other tumor killing factors, such as caspase 8 , TRAIL and Bax, and subsequently induces tumor specific apoptosis [19,18,20-23] and enhances the sensitivity of tumor cells to GCV without adverse effect. Thus, targeted gene therapy remains a highly promising system and progress in this field is gaining momentum.

An ideal targeted vector should have both good tumor specificity and high killing efficacy. To improve the killing efficacy, double suicide gene, dual-promoter, a promoter plus an enhancer and double enhancers, etc. have been employed. Kong et al [24] have exploited adenovirus-mediated TK/CD double suicide genes, which are more effective in killing breast cancer cells in vitro. Huang et al [25] have excised TK/CD suicide gene therapy with combination of radiotherapy to enhance radiosensitivity of tumor. Liao et al [26] have found that radiation can enhance therapeutic efficacy of hTERTpmediated gene therapy. But hTERT/CMV dual promoter vector can not increase the activity of the promoter due to possible interference between two promoters resulting in the decreased efficacy. CMV enhancer has been widely used to improve the suicide gene expression driven by hTERT promoter and has application potentials in targeted cancer gene therapy. Wang [11] has explored

Table 3 MCF-7 cell survival rates measured by MTT assay

\begin{tabular}{ll}
\hline Codes and Samples & Survival rates \\
\hline A. Cells without treatment & 1 \\
B. Cells transfected with pGL3-basic-EGFP and with GCV treatment & $0.987 \pm 0.006$ \\
C, Cells transfected with pGL3-basic-hTERTp-TK-EGFP-CMV and treated with GCV & $0.462 \pm 0.049^{*}$ \\
D. Cells transfected with pGL3-basic-hTERTp-TK-EGFP-CMV without GCV & $0.984 \pm 0.011$ \\
E. Cells transfected with pGL3-basic-hTERTp-TK-EGFP and treated with GCV & $0.515 \pm 0.025^{*}$ \\
\hline
\end{tabular}

Data are expressed as mean \pm standard deviation from three experiments. ${ }^{*}$ indicates $p<0.0001$ compared with other groups 

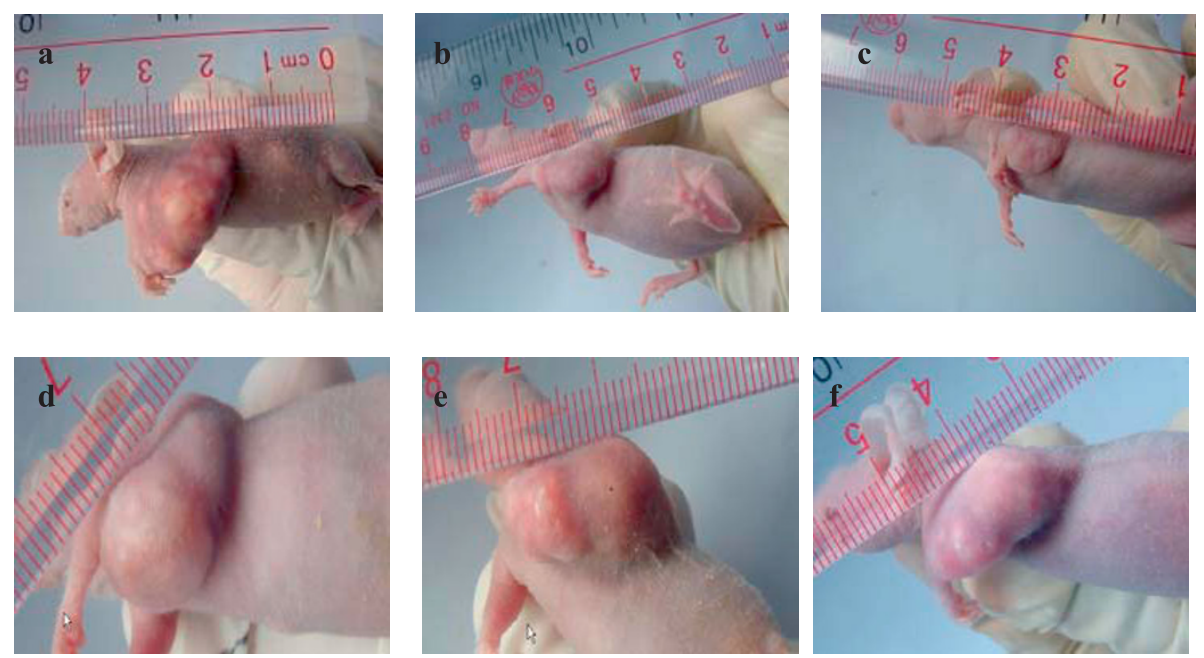

Figure 4 Tumor inhibition of pGL3-basic-hTERTp-TK-EGFP-CMV/GCV in nude mice with NPC xenograft. Shown are the NPC xenograft in nude mice without treatment (a), injected with GCV and the non-enhanced plasmid (b), injected with GCV and the enhance plasmid (c), injected with GCV(d), injected with Lipofectamine 2000 (e) and injected with the enhance plasmid without GCV (f).

the effects of hTERTp/CMV-regulated TK/CD system in five tumor cell lines and found that adding CMV enhancer increases TK/CD expression level by 3 26 times without affecting hTERTp-mediated targeting. Further study in HeLa cells [12] has revealed that enhancers can improve hTERT promoter activity by $6 \sim 13$ times, among which SV40-CMV dual enhancer/hTERT promoter has the highest activity, which is nearly 3 -fold of CMV enhancer/hTERT promoter; two hTERTp regulated $\mathrm{CD} / \mathrm{TK}$ fusion suicide gene driven by SV40/CMV dual enhancer has very high specificity and efficacy to tumor cells. Other vectors have also been used in cancer gene therapy. Song [27] has applied SB system, in which TK gene expression is targeted to cancer cells by hTERTp and enhanced by SV40 enhancer, to selectively kill liver cancer cells. In the present paper, TK was fused to EGFP for conventional observation of transfection efficiency. In addition, because hTERT only expressed in telomerase positive cells, stronger fluorescent signal reflect the relative expression of TK protein, indicating that we have successfully constructed a CMV enhanced-, hTERTp driven-TK gene expression vector,
pGL3-basic-hTERTp-TK-EGFP-CMV and increased the activity of hTERT promoter and expression of its downstream TK gene.

Our results indicate that transfection of the enhanced pGL3-basic-hTERTp-TK-EGFP-CMV with GCV treatment significantly inhibits the survival rate of nasopharyngeal carcinoma $5-8 \mathrm{~F}$ cells and the progress of nasopharyngeal xenograft in nude mice, and the enhanced pGL3-basic-hTERTp-TK-EGFP-CMV/GCV has much better tumor killing efficacy than pGL3-basichTERTp-TK-EGFP/GCV in both NPC 5-8F and MCF-7 cells. Quantitative fluorescence PCR showed that TK expression level was increased by 2 to 5 -fold in NPC 5$8 \mathrm{~F}$ and MCF-7 cells transfected with the enhanced vector compared with that in the cells transfected with non-enhanced vector. By contrast, TK expression was not altered by transfection of the enhanced vector in telomerase negative ECV cells. No side effects on liver and kidney by pathological examination were observed in in vivo experiment, suggesting the effects of the enhanced vector is specific to tumor cells and it is safe in in vivo application.

Table 4 Injection of pGL3-basic- hTERTp-TK- EGFP- CMV/GCV inhibited tumor development in vivo

\begin{tabular}{lllll}
\hline Sample & Animals & Tumor volume at day $\mathbf{3 9}\left(\mathbf{c m}^{\mathbf{3}}\right)$ & Tumor weight at day $\mathbf{3 9}(\mathbf{g})$ & $\underline{\text { Inhibition rate }}$ \\
\hline Blank & 5 & $6.23 \pm 0.04$ & $2.68 \pm 0.02$ & $/$ \\
Non-enhanced group & 5 & $3.51 \pm 0.02$ & $1.51 \pm 0.01$ & $43.68 \%^{*}$ \\
Enhanced group/GCV & 5 & $2.72 \pm 0.02$ & $1.17 \pm 0.01$ & $56.34 \%^{*}$ \\
\hline Enhanced group & 5 & $5.80 \pm 0.13$ & $2.51 \pm 0.05$ & $6.48 \%^{*}$ \\
\hline GCV group & 5 & $5.98 \pm 0.09$ & $2.56 \pm 0.09$ & $4.32 \%^{*}$ \\
\hline Lipofectamine group & 5 & $5.83 \pm 0.14$ & $2.51 \pm 0.02$ & $6.41 \%^{*}$ \\
\hline
\end{tabular}

Data are expressed as mean \pm standard deviation from three experiments. ${ }^{*}$ indicates $p<0.0001$ compared with the other group. 
The mechanism by which hTERTp/CMV-dual-regulated TK expression can enhance the targeted killing of nasopharyngeal carcinoma cells need to be further investigated. In our previous study on hTERT-TK expression vector, the killing effect of TK under hTERT promoter, which is a much weaker than CMV promoter, is significantly reduced compared with that of TK under the non-selective promoter CMV. In consistence with our other reports [7-9], our results suggest that addition of CMV promoter can significantly enhance TK efficacy without changing its targeting controlled by hTERT. Wang $[11,12]$ proposed that CMV can recognize specific binding sites of different activators, enhancers and promoters, therefore synergistically and dramatically promotes protein expression. In addition, co-effect of SV40 and CMV enhancers also enhance promoter activity because SV40 enhancer can effectively increase the amount of exogenous DNA in the nucleus. Therefore, the interference between hTERTp and CMV hindered the efficiency of vector.

In this study, we found that telomerase activities are significantly reduced in both NPC 5-8F and MCF-7 cells transfected with the enhanced vector after GCV treatment, but not changed in ECV cells transfected with the enhanced vector (Figure 4). One possible explanation is that the reduced telomerase activity in cells transfected with the enhanced vector is the result of the cell death induced by TK/GCV. We speculate that in the early stage of transfection of the enhanced vector, when GCV was not added into the cells, telomerase activity is temporally increased; after adding GCV into the cells, cell numbers dramatically decreased resulting in the reduced telomerase activity. However, we can not exclude other possibilities. Decreased telomerase activity has been shown to inhibit tumor proliferation. Transfection of eukaryotic vector containing antisense of hTERT in human gastric cancer SGC-7901 cells attenuated telomerase activity, reduced telomere length, decreased expressions of hTERT, bcL-2 and c-myC at mRNA and protein levels without changing hTR and TP1 expression, inhibited cell proliferation and arrested the cells in G0/G1 phase [28]. Injection of SGC-7901 cells transfected with the eukaryotic vector containing antisense of hTERT did not induce tumor development in nude mice, whereas injection of control cells without transfection induced touchable tumor growth. Transfection of hTERT small interfering RNA had similar results [29]. But it is more plausible that the mechanisms by which hTERT antisense or siRNA induced tumor apoptosis through reduced telomerase activity are different from that of the direct tumor killing of TK gene expression driven by hTERT promoter.

To our knowledge, the effect of TK gene expression driven by CMV enhancer/hTERT promoter has not been previously studied in NPC. In the present study, we also improved the methodology: 1) We applied coexpression of TK gene with EGFP in favor of the experimental observation, and adopted real time fluorescence quantitative PCR to measure the difference in TK gene expression; 2) We studied tumor-inhibitory effect of the enhanced vector both in vitro and in vivo, and explored the safety of in vivo application of the enhanced vector; 3) The enhanced vector had similar tumor inhibitory efficacy in both NPC and MCF-7 cells, suggesting it might have broader application potentials in other cancer therapies; 4) We compared the effect of the enhanced vector with the single promoter vector to elucidate the increased efficacy of the enhanced vector/ GCV system. As an enhanced targeting vector, transfection of pGL3-basic-hTERTp-TK-EGFP-CMV has obvious targeted killing efficacy on nasopharyngeal carcinoma and breast cancer, but its application in other tumor therapies need to be further investigated.

In conclusion, we successfully constructed the enhanced TK gene expression vector driven by hTERT promoter and CMV enhancer, and revealed that the enhanced vector indeed increased the TK expression and improved its killing efficacy on NPC in vitro and in vivo, indicating that the enhanced vector has clinical potentials in nasopharyngeal carcinoma gene therapy.

\section{Acknowledgements}

The study was supported by the Science and Technology fund of Guangdong Province (Project number: 2007B031003008).

\section{Authors' contributions}

CXS carried out the subtotal molecular genetic studies, participated in the design of the study, and performed the statistical analysis. ZW conceived of the study, and participated in its design and coordination. and drafted the manuscript. YHQ carried out the cell culture. SFM participated in the PCR $M T$, telomerase activity and DNA sequence. SFG participated in study work in vivo. All authors read and approved the final manuscript.

Competing interests

The authors declare that they have no competing interests.

Received: 14 April 2010 Accepted: 13 July 2010 Published: 13 July 2010

\section{References}

1. Wen Z, Xiao JY, Tang FQ, Tian Y, Zhao S, Chen B: The expression of telomerase and telomerase RNA in nasopharyngeal carcinoma (NPC) and $\mathrm{HNE}_{1}$ cell lines of NPC. Chinese Medical Journal 2000, 113:525-8.

2. Cheng RY, Yuen PW, Nicholls JM, Zheng Z, Wei W, Sham JS, Yang XH, Cao L, Huang DP, Tsao SW: Telomerase activation in nasopharyngeal carcinomas. Br J Cancer 1998, 77:456-60.

3. Wang YP, Tang XJ, Zhou QH, Che GW, Chen XH, Zhu DX: An experimental study on targeting suicide gene therapy for lung cancer with HSV-TK driven by hTERT promoter. Sichuan Da Xue Xue Bao Yi Xue Ban 2008, 39:701-5.

4. Zhang J, Wei F, Wang H, Li HM, Qui W, Ren PK, Chen XF, Huang Q: Potent anti-tumor activity of telomerase-dependent and HSV-1TK armed oncolytic adenovirus for non-small cell lung cancer in vitro and in vivo. J Exp Clin Cancer Res 2010, 29:52.

5. Zheng FQ, Xu Y, Yang RJ, Wu B, Tan XH, Qin YD, Zhang QW: Combination effect of oncolytic adenovirus therapy and herpes simplex virus 
thymidine kinase/ganciclovir in hepatic carcinoma animal models. Acta Pharmacol Sin 2009, 30:617-27.

6. Shen $Y$, Wang $Y$, Chen $S$, Xiao B, Su J, Tao Z: The effect of shRNA targeting hTERT on telomerase and the expression of PCNA and Caspase-3 in nasopharyngeal carcinoma cells. 2008, 22:411-5.

7. Wen Z, Xiao JY, Tian YQ, Chen BL: Down-regulation of telomerase and its RNA and apoptosis in HNE1 cellines of nasopharyngeal carcinama induced by hTR antisense oligonucleotide. International. J. Modern Cancer Therapy 2000, 3:77-81.

8. Mu SF, Wen Z, Guo MH, Xie MQ, Guan XF, Shen CX: TK gene targeted therapy mediated by the human telomerase promoter in nude mouse transplanted tumor of nasophyngeal carcinoma in vivo. Jie Fang Jun $Y_{i}$ Xue Za Zhi 2009, 34:155-8.

9. Xu F, Wen Z, Qiu YZ, Xiao JY: Tumor-specific TK gene therapy induced by hTERT promoter in human nasopharyngeal carcinoma (NPC) cells in vitro. Nan Fang Yi Ke Da Xue Xue Bao 2010, 30:695-9.

10. Guan XF, Wen Z, Shen CX, Mu SF, Zhang HZ, Xie MQ, Guo MH: Isolation and identification of tumor-like stem cells from human nasopharyngeal carcinoma cell line. Jie Fang Jun Yi Xue Za Zhi 2008, 33:1461-4.

11. Wang $L N$, Li Z, Xue ZG, et al: Incorporation of prokaryotic enhancer for enhancement of gene expression driven by hTERT promoter. Chin J Cancer Prev Treat 2006, 13:1125-30.

12. Wang $L N$, Zhang $Y K$, Zhou $Y$, et al: In vitro research of enhanced suicide gene vector for cervix cancer therapy. China Medical Engineering 2006, 14:567-75.

13. Zhou YB, Huang ZX, Ren CP, Zhu B, Yao KT: Screening and preliminary analysis of the apoptosis- and proliferation-related genes in nasopharyngeal carcinoma. Nan Fang Yi Ke Da Xue Xue Bao 2009, 29:645-7.

14. Li XP, Li G, Peng Y, Kung HF, Lin MC: Suppression of Epstein-Barr virusencoded latent membrane protein-1 by RNA interference inhibits the metastatic potential of nasopharyngeal carcinoma cell. Biochem Biophys Res Commun 2004, 315:212-8.

15. Zhang $H B$, Ren $C P$, Yang $X Y$, Wang L, Li H, Zhao M, Yang H, Yao KT: Identification of label-retaining cells in nasopharyngeal epithelia and nasopharyngeal carcinoma tissues. Histochem Cell Biol 2007, 127:347-54.

16. Wang J, Guo LP, Chen LZ, Zeng YX, Lu SH: Identification of cancer stem cell-like side population cells in human nasopharyngeal carcinoma cell line. Cancer Res 2007, 67:3716-24.

17. Marian CO, Shay JW: Prostate tumor-initiating cells: A new target for telomerase inhibition therapy? Biochim Biophys Acta 2009, 1792:289-96.

18. Takeda T, Inaba H, Yamazaki M, Kyo S, Miyamoto T, Suzuki S, Ehara T, Kakizawa T, Hara M, DeGroot LJ, Hashizume K: Tumor-specific gene therapy for undifferentiated thyroid carcinoma utilizing the telomerase reverse transcriptase promoter. JCEM 2003, 88:3531-8.

19. Song JS, Kim HP, Yoon WS, Lee KW, Kim MH, Kim KT, Kim HS, Kim YT: Adenovirus-mediated suicide gene therapy using the human telomerase catalyticsubunit (hTERT) gene promoter induced apoptosis of ovarian cancer cell line. Biosci Biotechnol Biochem 2003, 67:2344-50.

20. Gu J, Andreeff M, Roth JA, Fang B: hTERT promoter induces tumorspecific Bax gene expression and cell killing insyngenic mouse tumor model and prevents systemic toxicity. Gene Ther 2002, 9:30-7.

21. Komata T, Kondo $\mathrm{Y}$, Kanzawa T, Ito H, Hirohata S, Koga S, Sumiyoshi H, Takakura M, Inoue M, Barna BP, Germano IM, Kyo S, Kondo S: Caspase-8 gene therapy using the human telomerase reverse transcriptase promoter for malignant glioma cells. Hum Gene Ther 2002, 13:1015-25.

22. Bortolanza S, Qian C, Kramer MG, Gomar C, Prieto J, Farinati F, HernandezAlcoceba R: An oncolytic adenovirus controlled by a modified telomerase promoter is attenuated in telomerase-negative cells, but shows reduced activity in cancer cells. J Mol Med 2005, 83:736-47.

23. Jacob D, Davis J, Zhu H, Zhang L, Teraishi F, Wu S, Marini FC III, Fang B: Suppressing orthotopic pancreatic tumor growth with a fiber modified adenovector expressing the TRAIL gene from the human telomerase reverse transcriptase promoter. Clin Cancer Res 2004, 10:3535-41.

24. Kong $H$, Huang $Z H$, Li Q, Yang LC, Yu JL, Li Z: Adenovirus-mediated double suicide gene selectively kills breast cancer MCF-7 cells in vitro. Nan Fang Yi Ke Da Xue Xue Bao 2008, 28:907-10.

25. Huang SY, Zhang DS, Han JQ, Zhang N, Zhang SZ, Mu WL, Wei FC: Radiosensitization and anti-tumour effects of cytosine deaminase and thymidine kinase fusion suicide gene in human adenoid cystic carcinoma cells. J Int Med Res 2009, 37:479-90.
26. Liao ZK, Zhou FX, Luo ZG, Zhang WJ, Xiong J, Bao J, Han G, Zhang MS, Xie CH, Zhou YF: Radio-activation of hTERT promoter in larynx squamous carcinoma cells: an 'indirected-activator' strategy in radio-gene-therapy. Oncol Rep 2008, 19:281-6.

27. Song J, Kim C, Ochoa ER: Sleeping Beauty-mediated suicide gene therapy of hepatocellular carcinoma. Biosci Biotechnol Biochem 2009, 73:165-8.

28. Yang SM, Fang DC, Yang JL, Chen L, Luo YH, Liang GP: Antisense human telomerase reverse transcriptase could partially reverse malignant phenotypes of gastric carcinoma cell line in vitro. Eur J Cancer Prev 2008, 17:209-17

29. Shen Y, Zhang YW, Zhang ZX, Miao ZH, Ding J: hTERT-targeted RNA interference inhibits tumorigenicity and motility of HCT116 cells. Cancer Biol Ther 2008, 7:228-36.

doi:10.1186/1756-9966-29-94

Cite this article as: Shen et al.: Targeted gene therapy of nasopharyngeal cancer in vitro and in vivo by enhanced thymidine kinase expression driven by human TERT promoter and CMV enhancer. Journal of Experimental \& Clinical Cancer Research 2010 29:94.

\section{Submit your next manuscript to BioMed Central and take full advantage of:}

- Convenient online submission

- Thorough peer review

- No space constraints or color figure charges

- Immediate publication on acceptance

- Inclusion in PubMed, CAS, Scopus and Google Scholar

- Research which is freely available for redistribution
Biomed Central 